specific in children, with Erythema Migrans being the commonest presenting feature which is often missed as a heralding sign. Two children presented with LMN FP who we treated empirically for LD with Amoxicillin with subsequent results confirming the diagnosis. One was a holiday maker from an area not reported to have Lyme disease and the second child was on holiday in Republic of Ireland with known prevalence of Lyme disease. Both children presented to medical services in the preceding days with a rash and one with unilateral facial swelling (with development of LMN FP on same side). It is thought that up to fifty percent of facial Palsy in Children may be as a result of LD.

Conclusion Our findings confirm that LD is increasingly more common in the UK and should be considered as the most common cause of LMN FP. Symptomatic LD is potentially progressive with long term consequences. We propose empiric treatment of all cases of LMN FP with Amoxicillin or Doxycycline whilst awaiting serological confirmation of LD. In addition, clinicians should be considering LD much earlier in the differential of children presenting with a LMN FP.

\section{G323(P) A CASE OF SHINGLES WITH NO HISTORY OF CHICKEN POX}

A Butt, G Sinha. Paediatric Department, Walsall Manor Hospital, Walsall, UK

\subsection{6/archdischild-2018-rcpch.313}

Introduction Herpes Zoster Ophthalmicus (HZO) is a specific form of shingles that affects the dermatome of the ophthalmic nerve. It can have serious sequelae including permanent visual loss if there is ocular involvement.

Case report A 20-month-old child presented to the Paediatric Assessment Unit with a vesicular rash on his left forehead, and with swelling around the left eye. He had been treated with Flucloxacillin for impetigo, however this had failed to resolve the rash. He was apyrexial, passing urine and stool normally but had decreased oral intake. On examination the rash was restricted to the territory of the left ophthalmic nerve. The left eye was swollen with a yellow discharge and could not be opened. Swabs from the eye, and of the vesicular fluid were sent for bacterial culture and viral PCR. Initial bloods, including FBC and CRP, were normal, with no evidence of systemic infection. A diagnosis of $\mathrm{HZO}$ was made, based on the clinical findings, and the child was commenced on IV Aciclovir, IV Co-Amoxiclav, and Chloramphenicol eye drops. The following day the child had developed a fever and was tachycardic and tachypnoeic; the antibiotics were converted into Ceftriaxone. Varicella zoster $\operatorname{IgG}$ was found to be positive suggesting past infection. Ophthalmological opinion was sought to rule out ocular involvement. They found the child to be Hutchison's sign negative and agreed with the diagnosis of $\mathrm{HZO}$ without ocular involvement. The child received 7 days of Aciclovir, 7 days of antibiotics and 14 days of Chloramphenicol eye drops. The bacterial culture later isolated Moraxella catarrhalis, and the viral PCR confirmed Varicella zoster DNA.

Learning points Asymptomatic chickenpox is uncommon, although mild cases may be missed. Chickenpox infection can occur in utero and can cause scarring, neurological and ophthalmic complications, or the baby can be asymptomatic and present in early life with shingles. Accurate diagnosis is important to prompt early management. Examination of the eye with Ophthalmological input is important to rule out or manage ocular involvement. Hutchison's sign indicates nasociliary branch involvement increasing the likelihood of ocular sequelae.

\section{G324(P) CHILDHOOD STROKE DUE TO CEREBRAL ARTERIOPATHY; A PANDORA'S BOX?}

A Vatca, KE Maduemem, N McSweeney. Paediatrics, Cork University Hospital, Cork, Ireland

\subsection{6/archdischild-2018-rcpch.314}

Aim Stroke is an acute neurologic deficit that results from an ischaemic or haemorrhagic cerebral vascular event, persists for at least 24 hours, and results from a disturbance of the cerebral circulation. Childhood stroke is not common, but leads to significant morbidity and mortality. It is among the top ten causes of death in childhood. Stroke mimics are much commoner which make the diagnosis more challenging. We describe an intriguing case for which a wide array of tests has not revealed the precise aetiology.

Methods The clinical, laboratory and radiological data were reviewed.

Results A 3-year-old girl, only child of non-consanguineous Polish parents presented with marked altered level of consciousness preceded by upper respiratory tract infection. Examination revealed non-reactive pupils without focal neurological signs. Electroencephalography demonstrated diffuse encephalopathy. She was managed for probable encephalitis. There was clinical deterioration evident by headaches, an episode of focal seizure with subsequent right sided hemiparesis. Serial magnetic resonance image (MRI) and angiography (MRA) of the brain revealed diffuse abnormality of intracranial vessels with attenuated calibre of intracranial vessels and bilateral watershed infarcts. There was visual loss without evidence of optic neuritis. Metabolic, coagulation, autoimmune, infection, extracranial imaging and available genetic screen have been normal so far. Fluctuating blood pressure has remained a challenge in her management. She is being managed on immunosuppressive, antiepileptic, antihypertensive and antithrombotic agents. Multidisciplinary team follow-up is ongoing. Follow-up brain MRI and MRA (at five months) confirmed stable bilateral infarcts and vessels appearance.

Conclusion Cerebral arteriopathy has been recognised as a major risk factor for arterial ischaemic stroke in children. It may develop in a previously healthy child without any known risk factors. However, recent upper respiratory tract infection has been shown to be a significant predictor of underlying arteriopathy. ${ }^{1}$ The risk of recurrence in this case remains uncertain.

\section{REFERENCE}

1. Amlie-Lefond C, Bernard TJ, Sebire G, Friedman NR, Heyer GL, et al. Predictors of cerebral arteriopathy in children with arterial ischaemic stroke: Results of the international paediatric stroke study. Circulation 2009;119(10):1417-23.

\section{G325(P) AN EVALUATION OF OUR LOCAL PAEDIATRIC EPILEPSY SERVICES}

LB Patel, M El Gamal, D Murray, P Nair. General Paediatrics, East Lancashire Hospitals NHS Trust, Blackburn, UK

10.1136/archdischild-2018-rcpch.315 